
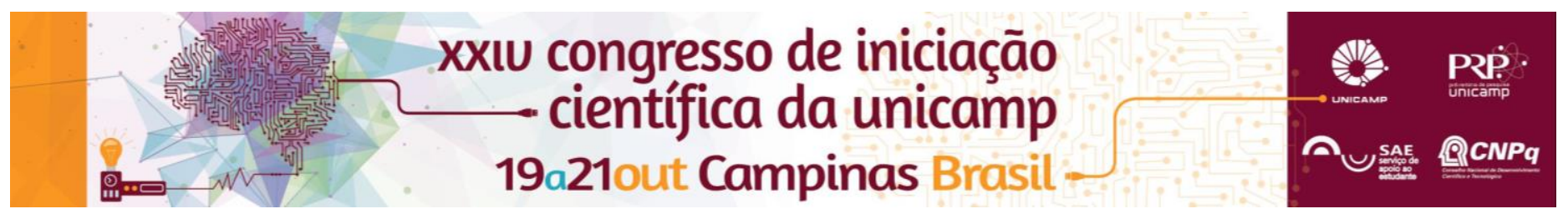

\title{
Biotransformation of Biomass Furan Derivatives
}

\author{
Luiz H. F. Araujo, Fábio D. Nasário, Paulo J. S. Moran, José A. R. Rosário
}

\begin{abstract}
Microorganisms were used to reduce regioselectively carbonyl groups in furan systems and the aplication of biocatalysis products in the production of new polymers (polyesters). The study had a great focus on the bioreduction of 5hydroxymethylfurfural (HMF) using yeasts available in the lab collection as well as assess its conversion efficiency.
\end{abstract}

\begin{abstract}
Key words:
Biocatalysis, bioreduction, biotransformation.
\end{abstract}

\section{Introduction}

The use of the lignocellulosic biomass to produce chemicals fuels has arisen as an interesting method to withdraw all the potential of the most used feedstock in fuels production. The main routes to obtain liquid fuels is from carbohydrates and they go through furan intermediates. Glucose is among the most used carbohydrate on this field and its conversion into liquid fuel passes through 5-hydroxymethylfurfural (HMF), a compound with a big and unexplored potential ${ }^{1-5}$. HMF can be oxidized into carboxylic acid and used to produce new polymers (polyesters). The chemical conversion procedures of HMF into commodities lacks of selectivity or demands non-mild reaction conditions and specific reagents. The use of microorganisms to reduce selectively the carbonyl group has arisen as in interesting alternative due to their regioselectivity, aqueous media reaction, wild conditions and quick conversions ${ }^{6-8}$.

\section{Results and Discussion}

The reduction of 5-methylfurfural (MF) and HMF employing $C$. albicans and $S$. cerevisiae displayed a quick and total conversion into their respective alcohols, 5methylfurfuryl alcohol (MFA) and 2,5bis(hydroxymethyl)furan (BHMF), taking about 1-2h. The conversion data were obtained by GC-MS.

The main challenge in the process was the extraction and purification of the product, which proved to be highly soluble in water, the biocatalysis media. The conventional use of a biphasic extraction with ethyl acetate and water was inefficient to separate the product. Firstly, polymeric resins were used to adsorb the product from the aqueous media, but their efficiency was not satisfactory. The most efficient resin was Supelite ${ }^{\mathrm{TM}}$ DAX-8, with $65 \%$. The other resins and their efficiency are showed on the Table 1. A good efficiency would be at least $75 \%$ for the products.

The second approach was an extraction by using deep eutectic solvent (DES), which is a system made up by choline chloride (a quaternary ammonium salt) and glycerol. Its action is based on the capability of its components in bonding with hydrogen donor compounds, which in that study would be the alcohol produced on the biocatalysis ${ }^{9}$. The GS-MS analysis indicated a reasonable amount of extracted product, but also traces of glycerol, one of the constituents of the DES. It was conducted a thin layer chromatography (TLC) of a BHMF and glycerol mixture in hexan:ethyl acetate (1:2), obtaining a $\mathrm{Rf}$ of 0,32 to BHMF and 0 to glycerol. Despite of the interesting $\mathrm{Rf}$ values, the attempt to separate the mixture on a silica gel chromatography column was not efficient, which made that extraction method unsuitable to the biocatalysis product (BHMF).

Table 1. Polymeric resins efficiency on the BHMF extraction on a BHMF $6,0 \mathrm{mg} / \mathrm{mL}$ aqueous solution.

\begin{tabular}{ccc}
\hline Resin & Mass $(\mathbf{g})$ & Efficiency \\
\hline \multirow{2}{*}{ Amberlite® XAD7HP } & $1,0 \mathrm{~g}$ & $44 \%$ \\
& $2,0 \mathrm{~g}$ & $50 \%$ \\
\multirow{2}{*}{ Supelite $^{\mathrm{TM}}$ DAX-8 } & $3,0 \mathrm{~g}$ & $61 \%$ \\
\hline \multirow{2}{*}{ Diaion® HP-2MG } & $2,0 \mathrm{~g}$ & $62 \%$ \\
& $3,0 \mathrm{~g}$ & $65 \%$ \\
\hline
\end{tabular}

\section{Conclusions}

The use of S. cerevisiae and C. albicans showed good results regarding the starting materials conversion (MF and HMF) into their alcohols, with total conversion, regioselectivity and short time. The reduction of HMF produced a high solubility compound, which decreased the extraction efficiency of the interest biocatalysis product. It had been tried two different ways for separating the product from the aqueous media, using polymeric resin and deep eutectic solvent. The first was not very promising and despite the second had extracted some of the product, the overall process became too long due to the glycerol removal need.

\section{Acknowledgement}

To FAPESP (process 2014/00108-9), PIBIC/CNPq and Institute of Chemistry UNICAMP.

${ }^{1}$ G. W. Huber, S. Ibarra, A. Corma, Chem. Rev., 2006, 106, 4044-4098

${ }^{2}$ A. Corma, O. de la Torre, M. Rens, ChemSusChem, 2011, 4, 1574-1577.

${ }^{3}$ G. W. Huber, J. N. Chheda, C. J. Barret, J. A. Dumersic, Science, 2005, 308, $1446-$ 1450

${ }^{4}$ A. V. Subrahmanyam, S. Thayumanavan, G. W. Huber, ChemSusChem, 2010, 3 , 1158-1161.

${ }^{5}$ D. M. Alonso, J. Q. Bond, J. A. Dumesic, Green Chem., 2010, 12, 1493-1513.

${ }^{6}$ Climent, M. J.; Corma, A.; Iborra, S. Green Chem. 2014, 16, 516.

${ }^{7}$ S. P. Teong, G. S. Yi, Y. G. Zhang, Grenn Chem., 2014, 16, 2015-2016.

${ }^{8}$ S. Dutta, S. De, B. Saha, ChemPlusChem, 2012, 77, 259-272

${ }^{9}$ Abbott, A. P.; Harris, R. C.; Ryder, K. S.; D'Agostino, C.; Gladden, L. F.; Mantle,

M. D. Green Chem. 2011, 13, 82 